\title{
LOS TRASTORNOS DEL ESPECTRO AUTISTA Y GENERALES DEL DESARROLLO: INCLUSIÓN SOCIAL Y CALIDAD DE VIDA ${ }^{1}$
}

\section{AUTISM SPECTRUM AND PERVASIVE DEVELOPMENTAL DISORDERS: SOCIAL INCLUSION AND QUALITY OF LIFE}

\author{
Manoel Baña \\ Universidad de la Coruña, España
}

Palabras clave: autismo, inclusión social, calidad de vida.

Keywords: autism, social inclusion, quality of life.

Los tres temas de esta conferencia son amplísimos, como lo muestra la cantidad de libros e investigación que sobre los mismos están apareciendo, ya sea de los trastornos del espectro autista y generales del desarrollo, ya sea de la inclusión social y educativa, ya sea de la calidad de vida. Voy a intentar hacer una presentación breve de nuestra experiencia para que se entienda de dónde parto y cómo llegué aquí. Mi atrevimiento a relacionarlos en tan poco tiempo es grande.

Hace unos 15 años nos reuníamos un grupo de padres/madres y profesionales para crear la primera asociación de padres. Nos reunía el interés y las ganas de que nuestros hijos o los hijos de los padres/madres que estaban allí, pudieran tener acceso a la vida, a la vida independiente, a la vida de calidad, a la vida digna y poder vivir como cualquier otra persona considerada "normal" o normotípica.

La cantidad de dudas que teníamos y confusiones era enorme. No sabíamos lo que nos esperaba, partíamos con mucha ilusión y también esperanza en conseguir lo que habíamos pensado y discutido. Pasaron 15 años, formamos un grupo numeroso de familias con la misma intención, mayor experiencia y la misma ilusión. Creamos otras dos asociaciones y una unidad clínica universitaria donde nos dedicamos a la investigación, la formación, la innovación y el desarrollo de vías de conocimiento para alcanzar nuestro objetivo inicial: la vida independiente y de calidad de nuestros hijos e hijas. En todo este caminar a este quien habla le ha tocado el rol de coordinador de todo este Proyecto y
Actividad. De esta forma, creamos un sistema de trabajo basado en sistemas de calidad sobre tres ejes: ética profesional (empatía y rigor), calidad de atención y calidad de vida. En este ámbito científico y profesional nos movemos cuando hablamos de calidad y sobre todo de calidad de vida y calidad de atención.

Uno de estos principios de calidad es la ética. Nos referimos, sobre todo a dos ejes vertebradores del ejercicio profesional: el rigor científico y/o profesional y la empatía. Mi exposición hace más referencia al rigor sin perder de vista el de empatía.

El Proyecto que iniciamos con las familias y las personas con TEA sigue avanzando con logros y, también, problemas y dificultades. Hemos conseguido muchos de los objetivos que nos planteábamos previamente, sobre todo con respecto a la normalización y la vida con los demás, las posibilidades de autonomía, la vida en las situaciones típicas de vida. Nuestra última finalidad sigue siendo su vida independientemente; han aparecido nuevas demandas y necesidades, las personas y las familias aprendieron a solicitar y exigir mayores prestaciones. De toda nuestra experiencia podemos señalar que lo que consideramos utopías en algún momento se convirtieron en realidad y estamos convencidos que podemos conseguir mucho más con respecto a la finalidad de una vida independiente y de calidad para las personas con TEA y/o TGD. Esta experiencia que me permite estar aquí me exigió conocer otro tipo de realidades, buscar otras formas de conocimiento, vidas, actividades. profesionales, 
padres y madres, organizaciones... con los que tuve la suerte de contactar, debatir, compartir e intercambiar nuestra información, posibilitando nuestro aprendizaje y la adopción de paradigmas y modelos contemporáneos y actuales. De ello surge la acepción del paradigma de apoyo, estableciendo y generando todo el sistema de apoyos que permita a cualquier persona desarrollar y realizar su vida de la mejor forma posible. Es lo que conocer por vida digna y calidad de vida.

Nuestra actividad nos llevó a creer más en las personas, en su capacidad de desarrollo y aprendizaje para su vida y la vida con los demás; para ello, tuvimos que superar algunas dificultades como las percepciones negativas y las connotaciones que hoy en día tiene el autismo para muchas personas y profesionales. Algunas de ellas hacen referencia a mitos de los años 30 y 40 del siglo pasado, cuando Leo Kanner los describía como personas que no daban cariño, que no tenían la posibilidad de dar cariño o no eran afectivos.

La realidad es que son personas muy afectivas, ingenuas, con una percepción sensorial extrema. Personas que pueden ejercer como auténticos maestros con los que aprender y con los que convivir de una forma rica y sorprendente, con una gran capacidad para luchar con las dificultades que van surgiendo en su vida diaria.

Uno de los problemas más grandes con que nos encontramos, es el tema de la sensibilidad y percepción social; esta sensibilidad que no les permite, muchas veces, desarrollarse de la mejor forma ni actuar convenientemente ya que se espera que tengan dificultades o sean incapaces de realizar actividades típicas de nuestra vida. Por ejemplo, es muy típico ver a un niño con TEA en un momento cualquiera moverse y llevar a cabo comportamientos repetitivos o inadaptados llamar la atención de una forma excesiva de las personas que lo rodean y mirar para él. El verse observado de una forma incomprensiva, incluso despectiva o sancionadora nos permite observar el descontrol que transmite el entorno a una persona que busca su comprensión. Supongo que a nadie le gusta sentirse el centro de atención de una forma extrema así como con miradas incomprensivas o sancionadoras, sea el contexto que sea (un aula, un pasillo, una plaza, una casa....). Así mismo, todos podemos comprender lo difícil de comportarnos si esperamos de cada comportamiento que uno emita, un juicio o un valor, sobre todo negativo o peyorativo. Es por ello que la sensibilización social, el cambio social debe ser prioritario en el establecimiento de ayudas para estas personas así como un objetivo prioritario para que estas personas puedan convivir y vivir con los demás. Lo relevante no es tanto si pueden o no convivir con los demás si no si se lo permitimos o posibilitamos; se supone que si tienen dificultades es porque necesitan ayuda.

Un ejemplo de ello son los padres/madres y profesionales que se acercan muchas veces con sus dudas sobre si estas personas pueden aprender y desarrollarse. Actualmente, Incluso profesionales que señalan que estas personas no son educables, no pueden educarse y desarrollarse intelectual, personal y socialmente. Hoy en día, esto no es discutible ni desde el punto de vista de la ciencia ni desde el punto de vista de la experiencia; todos los padres y madres, profesionales y demás personas cuando le dan la oportunidad de aprender se sorprenden de que aprendan. La ciencia actual lo presenta y defiende desde perspectivas tan diferentes como la biología a través de la Ley de Plasticidad Cerebral, la psicología y su teoría de la modificabilidad cognitiva, la neurología y el desarrollo neuronal, la fisiología y la configuración de los mapas cerebrales.

Desde esta perspectiva multidimensional de las ciencias y la educación en particular, sabemos que si posibilitamos la activación de los mecanismos adecuados para el aprendizaje, cualquier y toda persona aprende y se desarrolla. Lo más curioso con las Personas con Trastorno del Espectro Autista y General del Desarrollo, dentro del planteamiento general en que se desenvuelve este trabajo de la enseñanza y el aprendizaje, es que lo más general es que avanzan y aprenden más de los suponemos y esperamos. Hoy en día se suele hablar de las etapas de desarrollo que no se muestra pero sí suceden, no las vemos pero acontecen; quizás por ello, se llega a mencionar cada vez más como manifestaciones atípicas, desadaptadas o, frecuentemente, diferentes o diversas. Es frecuente observar saltos en el desarrollo y la aparición de nuevas demandas y necesidades que requieren de nuevos recursos en forma de ayudas $y$ apoyos educativos diversos y contingentes. 
También, se suele señalar que las personas con TEAS, tienen otro tipo de dificultades y otro tipo de demandas además de las típicas de las de su edad; en cuanto a su comunicación, en cuanto a sus manifestaciones difieren de las personas típicas o neurotípicas dado que su desarrollo es peculiar; no es que sean personas distintas de por sí si no de que son personas algo más distintas; es decir, iguales que los demás, diferentes como personas y algo más diferentes por sus necesidades en cuanto a sus características en el desarrollo y su alteración que determinan su forma de pensar y actuar, a mayores de sus diferencias y similitudes con todas las demás personas de su edad. Un ejemplo de ello es la necesidad de compartir con otras personas, las personas con Tea tienen esa necesidad, lo que sucede es que la manifiestan de forma particular; si la otra persona lo entiende y establece una comunicación le permitirá compartir, convivir y relacionarse; si no lo hace así, lo que posibilitará es que se frustre y con la experiencia frustrada se muestre indefensa con respecto a cada situación y/o contexto.

Si nos acercamos y vivimos con personas con TEAS y TGDS tenemos la posibilidad de establecer relaciones con ellas; si logramos comunicarnos es fácil entender y comprender lo que quieren, piden o solicitan; en esto son igual que cualquier otra persona; lo que los diferencia a mayores es en su forma de comunicarse con nosotros/as y es ahí donde necesitan de apoyo y ayuda. Una de las cosas que algunos nos señalan es que le exigimos a conocer nuestro mundo cuando muchos de nosotros/as no logramos entenderlo. También, nos señalan las múltiples contradicciones que cometemos y caemos así como en la falta de coherencia que acompaña a nuestro comportamiento y acciones; de ello, damos fé todos los días las personas que vivimos en él; por ello, no es fácil comprenderlo, sobre todo si uno es algo más diferente de los demás.

Hablar de trastornos del espectro autista y generales del desarrollo es hablar de vidas, de personas, de personas con calidad de vida. La calidad de vida entendida como una finalidad pero, también como un proceso y una forma de vida. La calidad de vida como proceso permite optimizar los procesos de aprendizaje y desarrollo. Si uno vive en calidad es más fácil hacer frente a los retos y dificultades que la vida le presenta todos y cada uno de los días que vive; esto se percibe de una forma muy clara cuando no se tiene; en los estados de desánimo, de desazón, de pérdida de calidad se observa lo difícil que es vivir y no digamos aprender y formarse; los contextos de calidad son importantísimos para la enseñanza y el aprendizaje.

Hablar de trastornos generalizados del desarrollo y del espectro autista es mencionar una serie de características que diferencian a algunas personas. Es como un segundo apellido donde el nombre es ser persona con todos los derechos y también deberes como tal, el primer apellido es ser diferente en cuanto a uno mismo con respecto a los demás y el segundo apellido es la diferenciación en cuanto a su alteración en el desarrollo. Como personas tienen derecho a la vida, la educación, el aprendizaje, el respecto de los demás, deberes en cuanto a su vida social y comunitaria, a sí mismo, a su familia y a su entorno físico y social. Como diferentes, tienen el derecho al respecto a la diferencia como Juan, Miguel, Ana, Sara... hijos de, vecinos, con su forma de pensar y sentir; en cuanto a TEAs y TGDs tienen el derecho a ser respetados en cuanto necesitan de apoyos y ayudas a mayores por sus necesidades educativas.

En este sentido, la investigación en desarroIlo y aprendizaje nos señala de su capacidad para aprender y desarrollarse como cualquier otra persona; así mismo, la Asociación Americana de Alteraciones del Desarrollo Intelectual (que edita una de las clasificaciones internacionales sobre discapacidad, reconocida por su prestigio y valía científica: el DSM) en su reunión de Salamanca en el 2002 concluye: la nueva definición de discapacidad intelectual hace referencia a las dificultades que el discapacitado tiene con respecto a su entorno no son debidas a él, con las derivadas del entorno que no le facilita su acceso y le obliga a comportarse de una forma poco adecuada a las necesidades que le genera. El cambio de perspectiva indica un cambio de análisis de las dificultades de los TEAS; si actuamos en función de ellos no le estamos proporcionando la ayuda para interpretar y adaptarse a su entorno; la ayuda debe ir en el sentido de adecuar y facilitar el entorno que le permite comprenderlo y desarrollar sus capacidades de una forma adecuada 
para resolver las dificultades que le origina y solucionar los conflictos que le plantea.

Otro argumento de peso en este argumento es el proporcionado por la teoría de la modificabilidad cognitiva; esta teoría señala que todas las personas tienen desarrollo y pueden tener más; su capacidad es modificable y depende de la estimulación que genere esta capacidad; asimismo, esta teoría defiende que no por más estimulación hay más desarrollo; se trata de adecuar la estimulación al potencial de aprendizaje y proporcionar su desarrollo. Se asienta en los principios de neuroplasticidad que señalan que las áreas cerebrales dañadas no pueden recuperarse pero sí sus funciones estimulando las áreas que no sufren daño y son adyacentes; para ello, lo adecuado es estimularlas para que generen las funciones de las áreas dañadas partiendo de la capacidad como señala Vygotski (1976); podemos concluir que el daño que pueda haber en un TEA se puede recuperar si se estimulan sus potenciales de aprendizaje, uno de los más percibidos y destacados en la investigación es el visual y sus funciones cognitivas de memorizar, recordar...estas estructuras de desarrollo pueden generarse y permitiendo a las personas un mayor conocimiento. En este sentido, podemos afirmar que no existe argumento convicente y contemporáneo que nos permita significar que la persona con TEA no pueda aprender y/o desarrollarse más y mucho más.

Ahora bien, cuando hablamos de trastorno general del desarrollo es conveniente mencionar que hacemos referencia a una alteración (por eso Trastorno) cualitativa (amplia, penetrante, generalizada) del desarrollo (aparece en la infancia). No podemos obviar que tienen una alteración del desarrollo que afecta a todo el, por eso tienen peculiaridades en cuanto al mismo (la investigación señala que los procesos sueles darse en distintas edades al típico y de distintas maneras). El desarrollo de los TEAS es un desarrollo peculiar y distinto al típico; algunas funciones aparecen más tempranas y otras más tarde que el desarrollo típico cronológico. Jerome Bruner (1978) habla de los procesos de aprendizaje y los procesos del desarrollo; señala Brunner que los procesos del desarrollo se dan en todas las personas de la misma manera y secuencia; así mismo, Bruner (1976) afirma que estos procesos suelen darse de forma espontánea y natural; cuando un proceso no se crea así tiene que respetarse su forma y estructura de cómo se originan; la dificultad reside en cómo crear los procesos de aprendizaje para posibilitar los de desarrollo, no es fácil aunque sí posible. Esto significa que los procesos de desarrollo en las personas con TEA no se dan de forma distinta a las personas típicas (estoy obviando el término de normal porque el término normal es un término muy confuso, de norma cuando en los seres humanos suele darse por mayoría, los que más coinciden guardan la norma); esto tiene grandes repercusiones en el desarrollo intelectual, sobre todo, el referido al cultural que depende del tercer factor del desarrollo: el social; los discapacitados poseen una gran dificultad: la posibilidad de establecer relaciones e interacciones sociales lo que les impide utilizar el tercer factor del desarrollo que nos permite adaptarnos al medio social y cultural en el que nos desenvolvemos; los TEA tiene un gran hándicap en este sentido y la posibilidad de poderlo superar es facilitarles un entorno social inclusivo, es decir, accesible y posible para ellos/as..

Es obvio que estas personas aprenden y se desarrollan; una de las conclusiones de las investigaciones contemporáneas es que los TEA y los TGD no son una enfermedad por lo que no pueden tratarse médica o clínicamente más que como otra persona; en este sentido, es importante delimitar lo que es debido a su alteración y lo que no es (pueden tener enfermedades, síntomas... como cualquier otra persona independiente de su trastorno). Como alteración del desarrollo hace referencia, sobre todo en cuanto a su caso más extremo (el trastorno autista) a una afectación de la comunicación social. En el DSM V se pone en evidencia esta connotación y se diferencia la comunicación que sí realizan los TEA y TGD y la dificultad para comunicarse con los otros y estos con ellos; es evidente que comunican pero su dificultad de hacernos entender con ellos en cuanto a lo que solicitan, demandan y necesitan es obvia. Su comunicación a través del comportamiento es importante por lo que atribuirles significados sociales a esos comportamientos forma parte relevante e imprescindible de toda atención e intervención con ellos/as. Si no es así, lo que sucede es su exclusión familiar, escolar, social.

Otra de las características típicas de los TEA y TGD es que parecen presentar un li- 
mitado repertorio de intereses y actividades; digo parecen porque da la sensación de que esta características obedece más a una imposibilidad por comprender y organizar el mundo que les rodea más a que no tengan interés; la sensación de indefensión que los absorbe hace que emitan comportamientos inadaptados más que debido a su alteración; la dificultad de utilizar el mundo físico para comunicarse se observa en su incipiente actividad con muchos de estos objetos a los que pierden interés por la falta de funcionalidad en esa comunicación; incluso, bastantes de estas personas suele presentar un inicio en la comunicación verbal que luego desaparece como consecuencia de su poca o inhabitual utilización. La dificultad en comprender la organización de su medio físico y social influye en su dificultad para relacionar los objetos, personas y situaciones lo que dificulta enormemente su funcionalidad y la funcionalidad de las mismas.

Para ello, pueden existir múltiples causas; se puede observar en la gran cantidad de hipótesis de trabajo y de investigación que a poco que se acerque uno a la literatura de esta temática podrá descubrir; podemos señalar la perspectiva biológica, la genética, la neurológica, la fisiológica... hipótesis como las neuronas espejo, el cerebro masculino extremo, las áreas cerebrales posiblemente dañadas, la investigación cromosómica donde observar la interrelación entre varios cromosomas, las de posibles virus, vacunas, enfermedades en la gestación, alteraciones hormonales,... nos hacen indicar la dificultad de su estudio así como un análisis detallado de sus causas y orígenes. Como resultado de esta gran variedad de hipótesis surge una considerable oferta de tratamientos, elucubraciones y ofertas de muchos tipos de terapia, incluso algunos peligrosos para la salud de la persona que los recibe. Es evidente que existe mucha literatura y también confusión en cuanto al tema, sin embargo lo que hoy tenemos claro es que como Trastorno no es una enfermedad y el único método seguro y adecuado es la educación. Lo que sí coincide la comunidad científica en general es de que existe un daño cerebral, localizado posiblemente en las áreas más profundas o internas del cerebro; algunas investigaciones hacen referencia a un daño en ciertas áreas de la corteza; la investigación fisiológica actual refiere a la gran activación de la corteza en cuanto a las personas típicas y en las mismas situaciones de estimulación o vida, lo que deshecha aquella hipótesis de investigación que señalaba que las personas con TEA y TGD no recibían estimulación o no se percibían su realidad. Hoy en día, si que evidenciamos que perciben su realidad y si aplicamos esta investigación tienen una gran dificultad para seleccionar la información, obviamente también, la tienen para organizarla y crear sus automatismos interfiriendo en su atención y aprendizaje

Por lo que se refiere a las hipótesis de trabajo en la psicología, podemos referirnos a Lorna Wing como la primera persona que se refiere de una forma amplia y concreta a las dificultades que presentan los TEA y TGD; se denomina hoy en día como la Triada de Wing. La tríada de Wing hace referencia a tres parámetros que aparecen en la forma más extrema de los TEA y TGD: el primero se conoce como Teoría de la Mente; su falta en los TEA determinó su conocimiento y dio lugar a su descubrimiento. Las personas con TEA y TGD suelen tener dificultades acerca de pensar con la mente de los otros y a pensar que el pensamiento de uno y otro es único lo que determina su falta de intención comunicativa porque si los dos sabemos y conocemos lo mismo para que tenemos que decírnoslo. Esto afecta al desarrollo en su conjunto a alterar la comunicación e interacción social. Investigaciones actuales ponen de manifiesto que no es una dificultad sola de las personas con TEA y TGD; es encontró que existen personas sin este trastorno, incluso en las que consideramos típicas que no tienen esta capacidad o no poseen Teoría de la Mente.

Un segundo parámetro (en el que podemos destacar a P.Hobson por sus investigaciones en él), un hace referencia a los sentimientos y es conocido como el de la Intersubjetividad; hace referencia a las emociones $y$, de forma general podemos hacer una aplicación del primer parámetro referido a lo intelectual o forma de pensar a este como personal o de forma de sentir; si uno siente como el otro o lo mismo que el otro para que expresar sus emociones y sentimientos; de ahí la característica que se dice de los TEA y TGD que no expresan sus emociones o no sienten de la misma forma que los típicos.

El tercer parámetro esta subdividido en dos, el denominado como de Coherencia Central (explicado así por U. Frith) que hace referen- 
cia a la dificultad que tienen los TEAS para entender el mundo, porque no son capaces de organizarlo ni de crear esquemas entendibles de él y el denominado como Función Ejecutiva por Ozonoff y Russell y que hace referencia a la dificultad de los TEA para organizarse y planificar. Si tienen dificultades para organizar su mundo y de organizar el mundo que más o menos perciben, su dificultad para aprenderlo y encontrarle sentido es evidente..

Estos parámetros que se conocen como la Tríada de de Wing son importantes para el diagnóstico; si se cumplen los tres en su justa medida podemos hablar del Trastorno Autista por excelencia. Si no se cumplen con todas y cada una de las características de la Tríada estaremos ampliando el espectro pasando de Trastorno Autista a Generalizados del Desarrollo y al Espectro Autista; en estos, la división o margen entre el Trastorno y no Trastorno está relacionado con la posibilidad y capacidad de una vida con los demás de forma adaptada y típica; en cuanto las dificultades afectan a la propia vida individual, social y comunitaria podemos hablar de Trastorno (todos somos obsesivos con algo, todos tenemos problemas de relaciones sociales, todos tenemos problemas de comportamientos repetitivos); es evidente que todos y todas tenemos características y singularidades con respecto a los indicadores de la Tríada, todos y todas tenemos dificultades en nuestra vida, algunas mayores y otras menores, en este sentido, Vygotski habla de la inteligencia como un eje no lineal si no quebrado, de capacidades y dificultades; en el caso de los trastornos este tipo de relación es más extremo: en los TEA suelen aparecer capacidades aumentadas y crecidas (lo visual y sus procesos) y dificultades grandes (relacionado con lo verbal, secuencial....)

Es por ello que vuelvo a incidir en que son personas iguales, diferentes y algo más diferentes; los TEAs y TGDs no deben ser una etiqueta, deben ser la descripción detallada de las necesidades y demandas para su ayuda y apoyo; es evidente que si les ayudamos a superar estas dificultades y solucionamos sus necesidades pueden aprender y desarrollarse como cualquiera otra persona. En este sentido de proporcionar ayuda debemos tener en cuenta lo que J. Bruner señala: los procesos de desarrollo y de aprendizaje cuando se realizan de forma normal son espontáneos y suceden de forma casi automática; cuando tenemos que crearlos aparece la dificultad de generarlos de forma que posibiliten su aprendizaje de esa forma espontánea y natural. Para que esto suceda así, Cole y Scrbiner (1982) señalan que es necesario que las personas participen y se sientan involucradas en su actividad de aprendizaje, participen en ella, vean las consecuencias de sus acciones y, sobre todo, vean la funcionalidad de su actividad y se sientan motivadas para ello. Esto podemos relacionarlo con lo que Whenmeyer (2006) denomina autodeterminación para lo que se necesita de un entorno inclusivo (que le permita participar y ser partícipe de él) y normalizado (que cumpla con las características comunes de la vida para la que aprende). La normalización porque para aprender a vivir en un entorno no existe otra posibilidad que estar en él, ver su organización, su función, el sentido de las normas, valores, actitudes... También porque las habilidades como sociales no tienen más posibilidad que ser entrenadas en el ámbito donde suceden y tienen sentido; esto es más importantes en el campo de las dificultades ya que si una persona tiene dificultades para organizar y entender su medio es evidente que si participa y forma parte de este entorno le será más fácil entenderlo. Asimismo, las investigaciones transculturales ponen de manifiesto la estrecha relación entre el desarrollo de los procesos intelectuales y le conjunto de actividades y acciones que se realizan en el ámbito y contexto cultural específico. Estos procesos se realizan en la experiencia diaria, real donde se entrenan las habilidades para ello (en el ámbito social es donde se percibe con mayor claridad: relacionarse y tener amigos, los hábitos de interacción, el desarrollo de las relaciones sociales, el diálogo, la discusión...). Todo eso por más que se les explique a las personas, si la persona no las lleva a cabo, no participan en ellas, si no las realizan, si no se encuentran en su situación difícilmente pueden entrenarlas, aprenderlas, encontrarles sentido...). Además, en el caso de los TEAS y la discapacidad en general si los aislamos de los estímulos de vida y sociales, de la vida de todos y todas, su dificultad irá en aumento y se hará mayor.

De estas posibilidades para que se sienta uno más y con los demás dependerá su vida futura, digna y de calidad y a ello hace referencia 
cuando hablamos de inclusión; si queremos que las personas vivan unas con otras tenemos que posibilitarles que aprendan a hacerlo, a adaptarse unas a otras, a vivir con, a solucionar sus conflictos de una forma racional, a establecer relaciones sanas, a ayudarse, a prestarse apoyo....

En este sentido, educar para la inclusión significa creer en las personas, que estas pueden aprender, darle posibilidades a pesar de que tengamos dudas en ellas. El primer paso para una educación inclusiva es partir de los intereses y características de las propias personas, no lo que deseemos o nos parezca si no de su realidad; una estrategia prioritaria en buscar una buena comunicación, entender y entenderse, buscar los niveles de comunicación óptimos que nos permitan establecer sistemas de ayuda y apoyo.

Con la inclusión hacemos referencia a la definición de la UNESCO cuando señala: una filosofía construida sobre la creencia de que todas las personas son iguales y deben ser respetadas y valoradas. Es una serie permanente de procesos en los cuales niños y adultos con dificultades tienen la oportunidad de participar plenamente en todas las actividades que se ofrecen a todas las personas. En el caso de los TEAS es contar con la persona, ofertar la ayuda y apoyo que necesita para el aprendizaje y desarrollo en su vida con los demás y entre ellos. Es darle la oportunidad de creer que e puede y tiene el derecho a decidir sobre su vida, a hacer, organizarla de la forma que crea conveniente, a decidir su futuro, a educarle en derechos y deberes, a darle la oportunidad de educarse para vivir con los demás, tener la posibilidad de una vida digna y de calidad y aportar su conocimiento para una vida mejor en todos y todos, para todos y todas. La inclusión exige ilusión, esperanza y compromiso. Asimismo, exige cierto riesgo, el de la improvisación que le permita adaptarse y sentirse incluido, ser capaz, sentir sus propias experiencias, aprender a su ritmo en un contexto de respecto, cariño y autoridad donde compartir alegrías y penas, aprender de la experiencia propia y la de los demás, equivocarse y erar para poder aprender de los propios errores y las experiencias de los mismos. Significa también desmitificar métodos, aprender a dar lo mejor de uno mismo, a mejorar con la experiencia de la vida de la educación, a analizar la propia experiencia, a evaluar para mejorar.

Una de las variables y factores que más inciden en la inclusión es el de la coordinación; este factor explica lo importante de la organización del aprendizaje para el desarrollo posibilitando que las personas y apoyos tengan un mismo lenguaje y estructura para la comprensión del mundo físico y social. Una variable muy presente en la atención a esta personas es la de la ilusión e interés, la confianza y el cariño que sienten y perciben de forma sensorial; en este sentido, el mejor método educativo para trabajar con TEAs sigue siendo aquel que tiene una mezcla de ilusión, transmite esperanza, creencia en la otra persona, seguridad en la dirección del aprendizaje y la educación además del interés por el propio trabajo y la persona que tenemos con nosotros/as.

Hablar de la inclusión es hacer mención a la calidad de vida. Con la inclusión queremos fomentar el desarrollo personal de la persona, el bienestar emocional, físico y material de la misma en el sentido de dotarle de posibilidades de controlar la propia vida; además, debemos proporcionarle la posibilidad de desarrollar la autodeterminación así como la toma de decisiones y observar y sufrir sus consecuencias. El aprendizaje de la autodeterminación está relacionado con la vida independiente ya que posibilitamos que las personas tomen sus decisiones y vean las consecuencias de las mismas; Whenmeyer (2006) señala que el desarrollo de esta capacidad no está relacionada directamente con el retraso mental; si está en la medda de que a mayor retraso más entrenamiento requiere. De esta forma, las personas se van dando cuenta de su capacidad de dirección de la propia vida y de la consecuencia de sus actos. Para la vida adulta y el aprendizaje de estas experiencias para nuestras vidas es importante porque en función de las consecuencias de nuestras acciones vamos aprendiendo su utilidad y función, incluso el fruto de nuestras actuaciones. Si no dejamos que los demás aprendan de las consecuencias de sus acciones no pueden aprender cuál es el fruto y cuál es el resultado de todas ellas por lo que decidir y tomar decisiones es una cuestión superficial para la propia vida en contra de lo que acontece realmente. Por ello, la autodeterminación es fundamental para la calidad de vida y se la exigimos a todas 
las personas con unos mínimos de madurez y adultez intelectual.

En este sentido un recurso importante para esta calidad de vida es la inclusión social; con ella hacemos referencia a la posibilidad que damos a las personas en situación de exclusión de poder aprender, relacionarse y convivir. La capacidad de vivir con otros en sociedad se desarrolla y aprende; muchas veces, olvidamos que estas estrategias tan relacionadas con los procesos psicológicos superiores tienen que entrenarse y activarse. Son las que hacen referencia a la solución de conflictos, toma de decisiones, comunicar y comunicarse, establecer relaciones sanas, enunciar una idea de forma coherente.... Todos estos procesos forman parte de la educación escolar por lo que tienen que ser educados, mejorados y optimizados pero necesitan de tiempo para ello y un espacio en lassituaciones de enseñanza y aprendizaje para modularlos, perfeccionarlos...En este sentido, desde hace unos años, se viene reivindicando la trasformación de las escuelas de forma que creen ciudadanos capaces para la sociedad del futuro; en ella tienen una especial cabida los formados como personas adultas, capaces, independientes y autodeterminadas. Una vida donde los valores de respecto, ayuda y apoyo deben ser troncales y formar parte de una sociedad con calidad de vida.

Los principios de calidad de vida deben ser un objetivo de la sociedad futura por lo que la educación debe de incorporarlos como prioritarios. En general, todos buscamos calidad de vida, esa vida que deseamos con recursos, satisfacción y logro persona. El modelo actual de calidad se centra y defiende que ésta es una dimensión que se exporta; frente al modelo tradicional de calidad de vida donde uno pensaba que renunciar a su vida era facilitar y mejorar la calidad del otro, el actual defiende que uno/a tiene que procurar calidad para transmitir calidad. La calidad de vida responde a ese conjunto de dimensiones donde un se muestra con logro, sintiéndose a gusto y lleno de energía; existen distintos modelos científicos de calidad; nosotros vamos centrarnos en el de Schalock y Verdugo, (2002) y el de familia (Summers y otros, 2005; Turnbull, 2006). Con ello queremos incorporar la noción como agente de cambio para la calidad de los TEAs y TGDs. En primer lugar, debemos centrarnos en los resultados personales elaborando programas y estrategias relacionadas con la persona y su desarrollo independiente de su alteración y capacidad. En segundo lugar, debemos abogar por principios y recursos dirigidos a buenas prácticas basadas en la calidad de trato y atención en función de las necesidades y demandas de las personas concretas. En tercer lugar, debe ser una apuesta por la mejora continua basada en la evaluación para la mejora de los servicios, apoyos y profesionales entre otros. En este sentido, cobra especial significación la mejora de los mecanismos sociales para la educación y el desarrollo empezando por la familia y la educación familiar, continuando por la escuela y la educación para la única sociedad que existe y la educación social, comunitaria y ciudadana.

El paradigma de apoyos es un buen recurso para conseguir esta calidad; nos permite ajustar los apoyos y ayudas a la frecuencia e intensidad de los mismos para alcanzarla; estos apoyos deben ser individualizados concentrándose tanto en las áreas de actividad, el potencial de aprendizaje como su realización y mejora continua. Todo ello tiene que ir acompañado de la perspectiva en la que la persona a quien va dirigido determina le programa y su desarrollo cuyo objetivo final sea la calidad de vida de la persona. Con TEA y TGD. En esta procura influye de una manera muy importante la percepción de los profesionales, de las personas cercanas, sobre todo cuando su falta de creencia en el cambio educativo y de aprendizaje se extrema; es lo derivado de aquellos profesionales de la educación que no le ven sentido a lo que enseñan y no perciben ningún beneficio en el alumno/a, los que derivan a estos alumnos a otras formas de formación o áreas ajenas a la educación escolar conocida como tal hoy en día. Whenmeyer y Garner (2003) señalan que más que la inteligencia es el entorno el que representa un impacto significativo para la autodeterminación y la calidad de vida; quizás, lo más importante no sea la inteligencia o discapacidad que se tenga si no que las personas con TEA y TGDs tengan garantizado un entorno en el que viven, aprenden, trabajan y juegan que les permita oportunidades para aprender y practicar las habilidades que les permitan ser dueños de su vida y decidir sobre ella, realizando elecciones, expresando preferencias y no dejar que las percepciones de quienes los rodean, expectativas y estereotipos les coarten 
las oportunidades para aprender y desarrollarse y llegar a ser agentes de sus propias vidas.

Con ello, pretendemos crear un contexto social inclusivo donde la persona con TEA y TGD tenga la oportunidad de aprender en un clima de calidad de vida donde las familias disfruten del tiempo juntos, tengan esperanza de futuro, buenas relaciones entre ellos y con la familia extensa y tengan relaciones sanas y cercanas donde abunde el diálogo y la comunicación. Deben ser el entorno donde se eduquen y desarrollen en valores y crezcan aprendiendo a ayudar en casa, a ser independientes, a tener tiempo para las necesidades de cada uno y a conocer las personas que están presentes en su vida (profesores, profesionales, amigos....) Asimismo, deben ser el enlace de la persona con el mundo social, su intérprete en él estableciendo nexos entre estas personas y su entorno próximo, estableciendo relaciones de ayuda mutua con los otros sistemas educativos (escolar, social...), sirviendo de enlace y coordinación entre los distintos servicios y procurando la mayor calidad de vida familiar posible

La calidad de vida debida hace mención a eso, a crear ambientes de vida adecuados, donde las personas estén a gusto, donde los momentos, los ambientes, los entornos de calidad de vida, fomenten tiempos de esperanza. Si cambiamos los entornos y los hacemos más cálidos para ellos es mucho más fácil que se sientan identificados, que no se bloqueen, que permitan trabajar con ellas y educarse, que se comuniquen, que aprendan a comunicarse. El fin u objetivo último es su vida futura, su vida independiente, su vida con los demás; los pro- gramas y proyectos centrados en la persona son el mejor recurso para ello; nos permiten centrarnos en sus necesidades y demandas, ajustar nuestra ayuda para que sea lo justa y necesaria posible, nos permite unificar criterios y finalidades en común a los distintos servicios educativos y es una herramienta sobre la que elaborar un Proyecto Conjunto y futuro de la persona sobre la que ir discerniendo las dificultades y consecuciones. Exige ser realistas y prácticos en su inicio y muy ambiciosos en sus objetivos finales (al fin y al cabo no existe investigación alguna que nos permita concluir sobre la imposibilidad de desarrollo y aprendizaje así como determinar los límites en cuanto a ellos).

A ello ayuda eliminado diferencias irreales como la capacidad y no, los capaces de los que no son... Este cambio lleva parejo uno de perspectiva, donde la realidad pueda ser distinta; las personas con TEAs y TGDs necesitan que creamos en ellos les ayudemos nos acerquemos y entendamos posibilitando que todo lo que tienen a su alrededor funcione lo mejor para ellos. Su enseñanza debe centrarse en sus capacidades posibilitando organización y estructura, respetando su ritmo de aprendizaje y posibilitando mejoras continuas.

Creo que merece la pena pelear por un mundo donde todos y todas podamos vivir, donde sentirnos útiles ayudando al otro/a. Muchas gracias por vuestra atención, espero que esto pueda ayudar algo más a las personas con TEAs y TGDs y al mismo tiempo nos permita ser mejores a todos y todas con el objetivo de una mejor vida de calidad y una sociedad más inclusiva y justa.

1 (N. de la Ed.). Conferencia dictada por el Dr. Manoel Baña el 16 de setiembre de 2011 en el marco de las XI Jornadas Internacionales de Psicología Educacional (revisada por el autor). Las mismas se han desarrollado en Montevideo, Uruguay y han sido organizadas por el Departamento de Psicología del Desarrollo y Educación de la Facultad de Psicología de la Universidad Católica del Uruguay.

Para citar este artículo:

Baña, M. (2011). Los trastornos del espectro autista y generales del desarrollo: inclusión social y calidad de vida. Ciencias Psicológicas V (2): 163-191. 